EUROPEAN ORGANIZATION FOR NUCLEAR RESEARCH

CERN-EP-2000-011

January 21, 2000

\title{
A liquid scintillator detector with wavelength-shifting fibre readout
}

\author{
M. Doucet ${ }^{a}$, J.-P. Fabre ${ }^{a}$, G. Grégoire ${ }^{b}$ J. Panman ${ }^{a}$, P. Zucchelli ${ }^{a, c}$ \\ ${ }^{a}$ CERN, Geneva, Switzerland \\ ${ }^{b}$ Université Catholique de Louvain, Louvain-la-Neuve, Belgium \\ ${ }^{c}$ On leave of absence from INFN - Sezione di Ferrara
}

\begin{abstract}
A technique based on liquid scintillator with wavelength-shifting fibre readout is interesting for large-mass neutrino detectors. In this paper, we present the first results obtained with a laboratory prototype assembled at CERN. In the configuration tested, a large light yield has been observed and attenuation lengths of the order of $3.2 \mathrm{~m}$ have been measured. As a comparison, we also measured the attenuation length of the fibres in air using the same setup. No significant difference in attenuation length between the fibres in air and in liquid was measured.
\end{abstract}

(To be submitted to Nucl. Instr. and Meth.) 


\section{Introduction}

The study of neutrino interactions calls for large massive detectors. These detectors generally use a small number of detector techniques. Consequently, they can be made uniform and simple in design. Detectors made of tubes filled with liquid scintillator read by wavelength-shifting (WLS) fibres have been identified as a promising solution for large scale neutrino experiments $[1,2]$. A single container of liquid scintillator segmented by optical separators can provide a granularity of a few centimetres over a large volume. This volume can be read out by WLS fibres, hence reducing the surface to be equipped with photo-detectors. If one can in addition make the tubes very long, the number of photosensitive devices necessary to read the volume of the detector can be reduced as the detector is made wider. The maximal length of the tubes is dominated by the attenuation length of the WLS fibres and the minimal number of photo-electrons that need to be detected to perform the physics measurements. It is therefore important to study the attenuation length of WLS fibres in conditions similar to this experimental environment.

In this paper we describe tests of a detector based on liquid scintillator and WLS fibres. We present the results of attenuation length measurements performed with two test tubes of $4 \mathrm{~m}$ in length. The goal of this study is twofold. First we want to measure the attenuation length in conditions similar to a real detector, and second we want to compare the attenuation length measured in these conditions to the attenuation length of the same fibres in air. Since the refractive index of the liquid scintillator $(\mathrm{n} \sim 1.566)$ is larger than that of the outer cladding of the WLS fibre $(\mathrm{n} \sim 1.42)$, we expect to lose the light that would be caught in the outer cladding would the fibre be placed in air. The light caught in the cladding has a shorter attenuation length than the light travelling through the core due to the quality of the interface between the outer cladding and the surrounding medium, which is subject to deteriorations caused by the handling of the fibre. Since the light caught in the cladding can be larger [3] than the light caught in the core, we want to verify whether the attenuation length of the fibre when immersed in the liquid is different from its attenuation length in air. A study of the light yield with different liquids and different reflective surfaces will be described in a forthcoming publication.

\section{$2 \quad$ Experimental setup}

Figure 1 shows a sketch of the experimental setup. Two aluminium tubes of $4 \mathrm{~m}$ in length were used. One of them had an outer cross-section of $3 \times 3 \mathrm{~cm}^{2}$ and was painted on the inside surface with Bicron $\mathrm{TiO}_{2}$ reflective paint [4]. The aluminium had a thickness of $3 \mathrm{~mm}$, so that the cross-section of the volume of the liquid filling the tube was $2.4 \times 2.4 \mathrm{~cm}^{2}$. The second tube had an outer cross-section of $4 \times 4 \mathrm{~cm}^{2}$ and an inner cross-section of $3.4 \times 3.4 \mathrm{~cm}^{2}$. Its inner aluminium surface was cleaned by chemical 


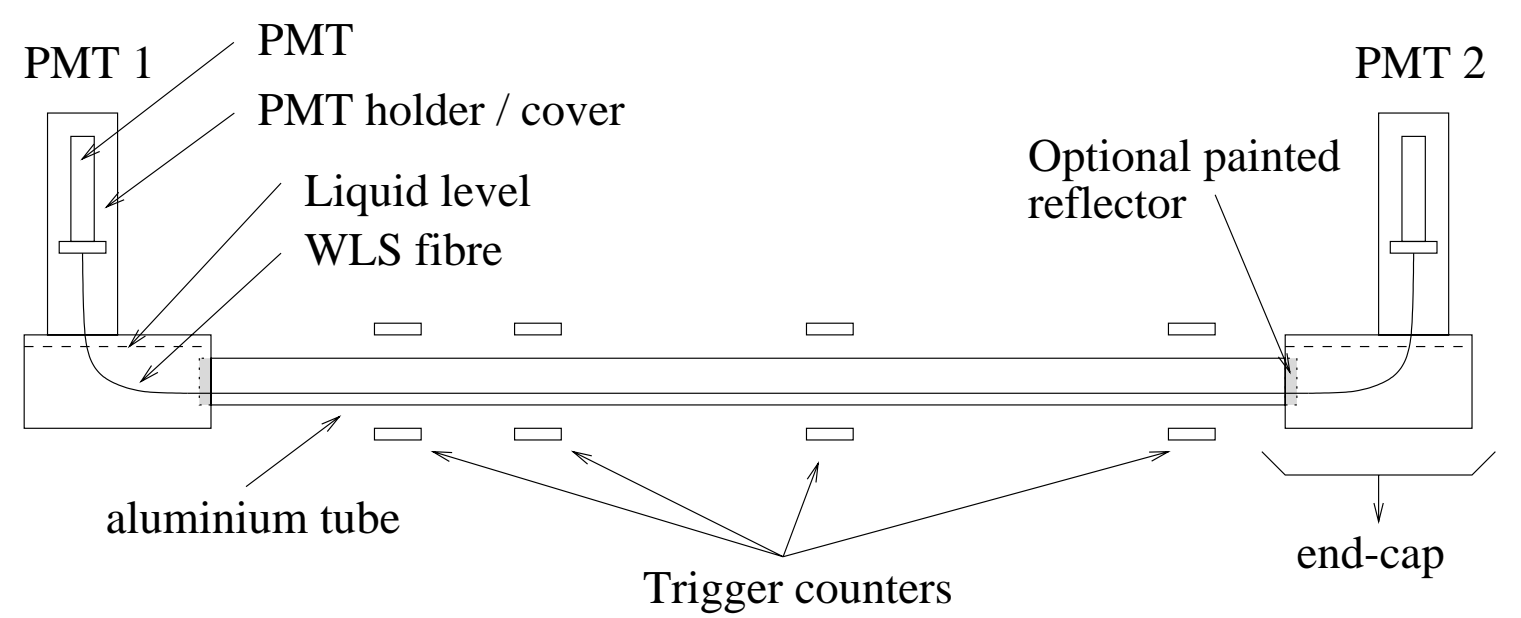

Figure 1: Schematic view of the experimental setup. The fibre runs through the centre of the tube, lying on the lower surface. The setup can be used with aluminium tubes of any given length.

passivation in order to provide a uniform reflective surface. This second tube was used to exclude the possibility that any feature was introduced by some inhomogeneity of the tube with the reflective paint.

The tubes were placed consecutively in a common setup which held them on each end by a light-tight end-cap anodized on the inside with a black coating. Each endcap held a photomultiplier tube (PMT) in a vertical position. The PMTs used were Hamamatsu R647P with bialkali photocathode. The quantum efficiency of this PMT is about $10 \%$ at $550 \mathrm{~nm}$. The ends of the tube in the setup could be left open to the end-caps, or closed by an aluminium wall painted with reflective paint. A Kuraray Y11-MSJ200 [5] fibre with a diameter of $1.2 \mathrm{~mm}$ was placed inside the tube. The polished ends of the fibre were applied to the window of a PMT by a teflon cap. To reach the PMTs, the ends of the fibre extended about $30 \mathrm{~cm}$ from the tube on each side and were bent with a radius of curvature of about $10 \mathrm{~cm}$. The fibre was placed at the bottom of the tube to stabilize its position. The light collection depends on the position of the fibre within the aluminium tube. A Monte Carlo simulation has shown that a fibre placed against the wall of the tube can be expected to collect up to $20 \%$ more light than when it is placed exactly at the centre of the tube [6]. The apparatus was filled with Eljen EJ-399-04 scintillator [7], such that the tube was completely filled. This scintillator is based on economical and safe commercial components (LAB [8] and PXE [9]) and does not attack the fibres. We tested that the quality of the fibres did not degrade throughout data acquisition periods of up to about two months.

The tests were performed with cosmic-ray muons. For the trigger, four identical pairs of scintillator pads read by 1.5 inch PMTs were placed along the tube. For each pair, the pads were placed above and below the tube to provide a cosmic-ray trigger. The distances between the pads and the centre of the tube were $6.5 \mathrm{~cm}$ (above) and 
$5.5 \mathrm{~cm}$ (below). The scintillator pads of the trigger system had a surface of about $5 \mathrm{~cm} \times 2 \mathrm{~cm}$ and a thickness of $5 \mathrm{~mm}$. For each cosmic trigger, the signal of each of the ten PMTs (two for the fibre and $2 \times 4$ for the trigger counters) was read. The trigger pairs were moved along the tube to scan its length. All PMT signals were read by means of Lecroy 1182 VME ADCs. The VME crate was connected to a desktop PC by optical fibres through a Bit3-618 PCI-VME interface [10]. The trigger rate of the setup was 2-3 counts per minute per scintillator pad pair. The response of the PMTs was between 8 and 9 ADC counts per photo-electron. With this setup, using the tube painted with the reflective paint, a mean light yield of about 20 photo-electrons was collected by each PMT for muons crossing the middle of the tube.

Five configurations of the setup were used:

- Configuration 1: The fibre was placed in the $3 \times 3 \mathrm{~cm}^{2}$ tube painted with reflective paint. The ends of the tube were closed by reflective walls.

- Configuration 2: The fibre was placed in the $3 \times 3 \mathrm{~cm}^{2}$ tube painted with reflective paint. The ends of the tube were open to the black end-caps. The data taken with this configuration were compared to data taken in Configuration 1 with the same fibre.

- Configuration 3: The fibre was placed in the $3 \times 3 \mathrm{~cm}^{2}$ tube painted with reflective paint, in a transparent teflon tube so that the liquid did not touch the fibre. The ends of the tube were open to the black end-caps. The same fibre as in Configuration 1 was used.

- Configuration 4: The fibre was placed in the $4 \times 4 \mathrm{~cm}^{2}$ aluminium tube. The ends of the tube were open to the black end-caps. A new fibre was used.

- Configuration 5: The fibre was placed in the $4 \times 4 \mathrm{~cm}^{2}$ aluminium tube, in a transparent teflon tube so that the liquid did not touch the fibre. The ends of the tube were open to the black end-caps. The same fibre as in Configuration 4 was used.

Configurations 1 and 3 were our main configurations for this series of measurements. With these two configurations it was possible to measure the attenuation length of the fibre in liquid (Configuration 1) and in air (Configuration 3) without having to change the fibre or modify the setup extensively. These measurements provide the results on which we draw the conclusions of this paper. The additional measurements with the other configurations were done to have a better understanding of the light collection process and of the different features of the results.

Because the light collection can take place over a distance of several centimetres, some light can be lost in the end-caps for measurements near the edges of the tube if these are not closed by reflective walls. We compared the results obtained with Configuration 1 to measurements performed using Configuration 2 to study these effects. 
Local variations in the light yield can be introduced if the reflectivity of the inner surface is not uniform along the length of the tube. This can happen if the coating of reflective paint is not the same everywhere. To cross-check our measurements, we used the second tube described above (Configurations 4 and 5), which has a uniform aluminium inner reflective surface.

\section{Measurements}

Series of measurements were done for each of the five configurations described above. The light yield was measured on both sides of the fibre by the photon-counting PMTs. Examples of signal distributions are shown in figure 2. Pedestal subtraction has been applied to these distributions. The pedestal for each PMT was measured in an independent run. The data shown in figure 2 were taken in Configuration 1, with tracks passing at a distance of $340 \mathrm{~cm}$ from PMT 1. The average light yield was measured by fitting a gaussian to the distribution of the logarithm of the ADC counts, which approximates the shape of a Landau distribution.

To be able to use the transparent teflon tube, the ends of the aluminium tube were open to the end-caps. To study the effect of the light lost near the edges of an open tube, we compared to data taken with Configuration 2. Figure 3a shows the geometric average $^{1}$ of the mean signals close to one end of the $3 \times 3 \mathrm{~cm}^{2}$ tube painted with reflective paint. Both data taken with open and closed ends are shown (Configurations 1 and 2). The measured edge effect is small. There is a more pronounced fall in the light yield in the first $20 \mathrm{~cm}$ near the edge when the end is open than when it is closed. Figure 3b shows data taken with the $4 \times 4 \mathrm{~cm}^{2}$ tube with the aluminium inner surface (Configuration 4). We see that the geometric average measured with open ends on a tube with an aluminium inner surface is more constant than in the case of the painted tube. This behaviour can be explained by the fact that a smaller number of reflections occur with the aluminium reflector, so that the light collection takes place at closer range. In the following measurements, only the data with open ends taken at a distance of at least $20 \mathrm{~cm}$ from the ends of the tube were used.

Figure 4 shows the results obtained with Configuration 1. Figures $4 \mathrm{a}$ and $\mathrm{b}$ show the average number of ADC counts measured with the PMTs on both sides of the tube. The errors on the points include both statistical and systematic errors. The systematic errors on the points were evaluated from the geometric average of the mean signals measured on the two PMTs. Figure 4d shows the geometric average of the two PMTs as a function of the distance to one side of the tube. The mean residual with respect to the mean value of the geometric average was taken as a systematic error on the ADC counts. This error is about $10 \%$. Figure $4 \mathrm{c}$ shows the ratio of the average number of counts on the two PMTs (figures $4 \mathrm{a}$ and $\mathrm{b}$ ) as a function of the distance from one side of the tube.

\footnotetext{
${ }^{1}$ The geometric average of the signals of the two PMTs is defined as $\sqrt{\text { signal } 1 \times \text { signal } 2}$.
} 
The data samples of Configuration 1 presented in figures $4 \mathrm{a}, \mathrm{b}$ and $\mathrm{c}$ were independently fitted to an exponential to measure the attenuation length. The results of the fits are presented on the relevant figures, and are reported in table 1. They are consistent with a single component of attenuation, meaning that the short attenuation length component of the cladding light is small compared to the core light, which is what we expect if the fibre is immersed in the liquid.

Similar sets of data were taken with the fibre in air (Configuration 3) and are presented in figure 5. We measure a smaller geometric average than in Configuration 1, due to absorption in the teflon tube. The emission spectrum of the wavelength-shifter is known not to be affected by the incident light spectrum, which could be different with and without the teflon tube. Apart from the light yield, the results are similar to those of Configuration 1. The fitted values of the attenuation length are reported in table 1. The data are consistent with a single component within the uncertainties on the measurements. We also expect an additional component of shorter attenuation length due to the cladding-air interface. This component can become very small relative to the core component after the $50 \mathrm{~cm}$ of fibre between the PMTs and the first measurement points, and it is not seen in our data.

The results of the measurements performed with the tube with the aluminium inner surface and the fibre directly in the liquid (Configuration 4) are presented in figure 6 . The results of the measurements performed with the teflon tube (Configuration 5) are presented in figure 7 . These results are consistent with the measurements made with the painted tube. No effect due to inhomogeneities of the painted tube was detected. The attenuation length values obtained from the fits of these data are reported in table 1. It is interesting to note that the geometric average of the two PMTs is about a factor 2.5 lower for the aluminium reflector than with the reflective paint in otherwise similar conditions. From pure geometric considerations, we would expect more light with the aluminium tube, which has a larger width. This confirms that the paint provides a better reflective surface for our detector.

Table 1 shows all the attenuation length measurements with their statistical and systematic errors. The systematic errors reflect the reproducibility of the attenuation length measurements. These errors include:

1. Errors from the light yield: As described above, the measurements at different positions were assigned a systematic error to reflect the variation of the light yield as a function of the position. This effect was about 10\% (see description above).

2. Errors from the pedestal position: The positions of the pedestals were moved up and down by the width of the pedestal distribution. The effect of a shift on the position of the pedestal is larger when the signal is weak, as in Configurations 3 to 5 . The systematic uncertainty can be as large as $\pm 10 \mathrm{~cm}$ in attenuation length 
for one of the PMTs (PMT 1). The systematic uncertainty was of the order of a few centimetres for the other PMT (PMT 2), which was found to be generally less noisy.

3. Errors from the fit: In the cases where the light yield was low, the ADC count distribution extends down to the pedestal. In this region the distribution is less reliable. To avoid any problem, the region near the pedestal was not used in the fit. The fit region was varied to evaluate the stability of the procedure. The resulting systematic error on the attenuation length can be as large as $30 \mathrm{~cm}$ for weak signals like those given by Configuration 5 .

Figure 8 shows the attenuation length measurements obtained with the different configurations. The values measured with the different configurations are consistent. In particular, the attenuation length of the fibre placed in air is compatible with the attenuation length measured with the fibre in the liquid. We observe no significant difference in terms of attenuation length between the results obtained with the tube with the aluminium inner surface and the tube with the reflective paint.

Table 1: Attenuation length of the fibre measured in different conditions. The first error is statistical and the second is systematic.

\begin{tabular}{|l|l|l|}
\hline Configuration & Measurement & Attenuation length $[\mathrm{cm}]$ \\
\hline \hline 1 (liquid) & PMT 1 & $299 \pm 21 \pm 12$ \\
1 (liquid) & PMT 2 & $340 \pm 25 \pm 5$ \\
1 (liquid) & PMT 1 / PMT 2 & $324 \pm 14 \pm 5$ \\
\hline 3 (air) & PMT 1 & $293 \pm 15 \pm 18$ \\
3 (air) & PMT 2 & $321 \pm 16 \pm 12$ \\
3 (air) & PMT 1 / PMT 2 & $310 \pm 11 \pm 14$ \\
\hline 4 (liquid) & PMT 1 & $285 \pm 13 \pm 17$ \\
4 (liquid) & PMT 2 & $351 \pm 21 \pm 23$ \\
4 (liquid) & PMT 1 / PMT 2 & $321 \pm 11 \pm 17$ \\
\hline 5 (air) & PMT 1 & $284 \pm 13 \pm 18$ \\
5 (air) & PMT 2 & $370 \pm 23 \pm 24$ \\
5 (air) & PMT 1 / PMT 2 & $322 \pm 16 \pm 38$ \\
\hline
\end{tabular}

\section{Conclusions}

We demonstrated the feasibility of a liquid scintillator detector read out by wavelengthshifting fibres. Using this detector, we studied the attenuation length of the fibres and found that the attenuation length of a fibre in air is compatible with the attenuation 
length of the same fibre in liquid scintillator, even though we expect a greater component of cladding light when the fibre is placed in air. The measured value of the attenuation length of the fibre in a tube painted on the inside with reflective paint (with the fibre directly in the liquid) is $\lambda_{\text {liquid }}=324 \pm 14 \pm 5 \mathrm{~cm}$. This value was obtained using the ratio of the PMTs on each side, which provides more robust measurements. The results obtained with the painted tube were checked with a tube with aluminium surfaces. No uniformity problem was found with the painted tube.

The light outputs measured using the tube with the reflective paint and using the tube with the aluminium reflector are significant and support the use of this detector technique. The tube with the aluminium reflective surface had a light yield 2.5 times lower than the painted tube. The measured attenuation length would limit the length of the detector elements to about $4 \mathrm{~m}$ unless optimized wavelength-shifting fibres are used. The compatibility between the fibre and the liquid scintillator was tested for periods of about two months and no incompatibility was discovered.

\section{Acknowledgements}

We would like to thank Daniel Bourillot, Jean Dupraz, Georges Roiron and Rudolf Buhler for their indispensable help with the test setup.

\section{References}

[1] The MINOS Collaboration, "The MINOS Liquid Scintillator Detector", NuMi Note L292 (1997).

[2] M. Doucet, J. Panman and P. Zucchelli, CERN-OPEN-99-010 (hep-ex/9905029).

[3] N.A. Amos, A.D. Bross and M.C. Lundin, Nucl. Inst. and Meth., A297 (1990) 396-403.

[4] Bicron, Newbury, Ohio, USA. BC-622A reflector paint.

[5] Kuraray Co., Ltd., Tokyo, Japan.

[6] P. Vilain and G. Wilquet, private communication.

[7] Eljen Technology, Sweetwater, TX, USA. EJ-399-04 scintillator.

[8] Eljen Technology, Sweetwater, TX, USA. Linear Alkylbenzene.

[9] Kock Chemical Company, Corpus Christi, TX, USA.

[10] SBS Technologies, Inc., Albuquerque, NM, USA. 

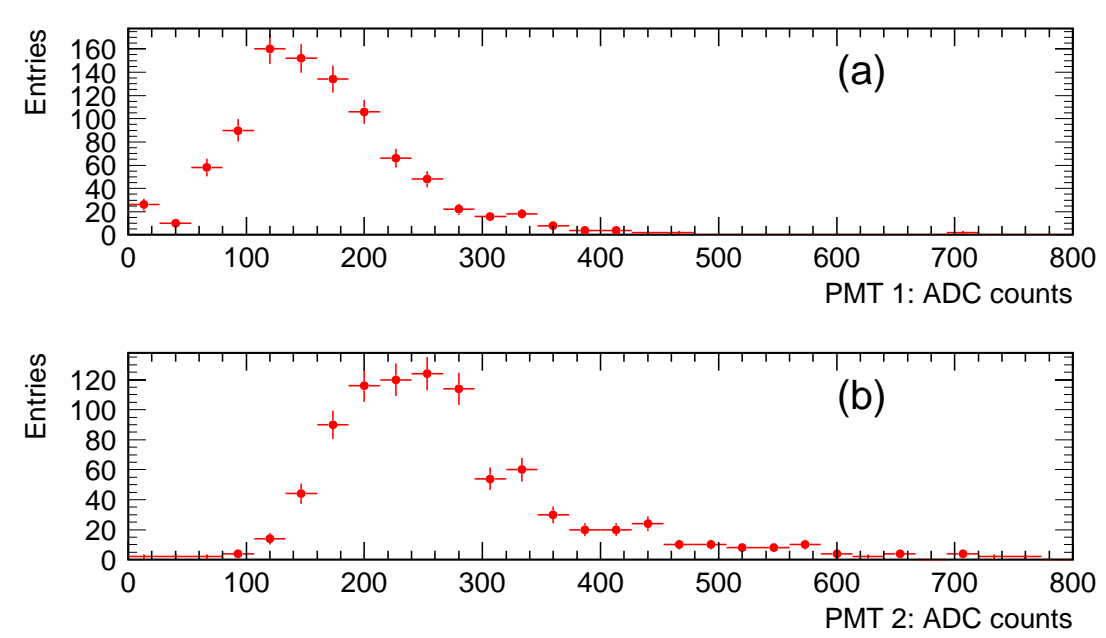

Figure 2: Example of measured ADC count distributions from PMT 1 (a) and PMT 2 (b). These data were taken at $340 \mathrm{~cm}$ from PMT 1.
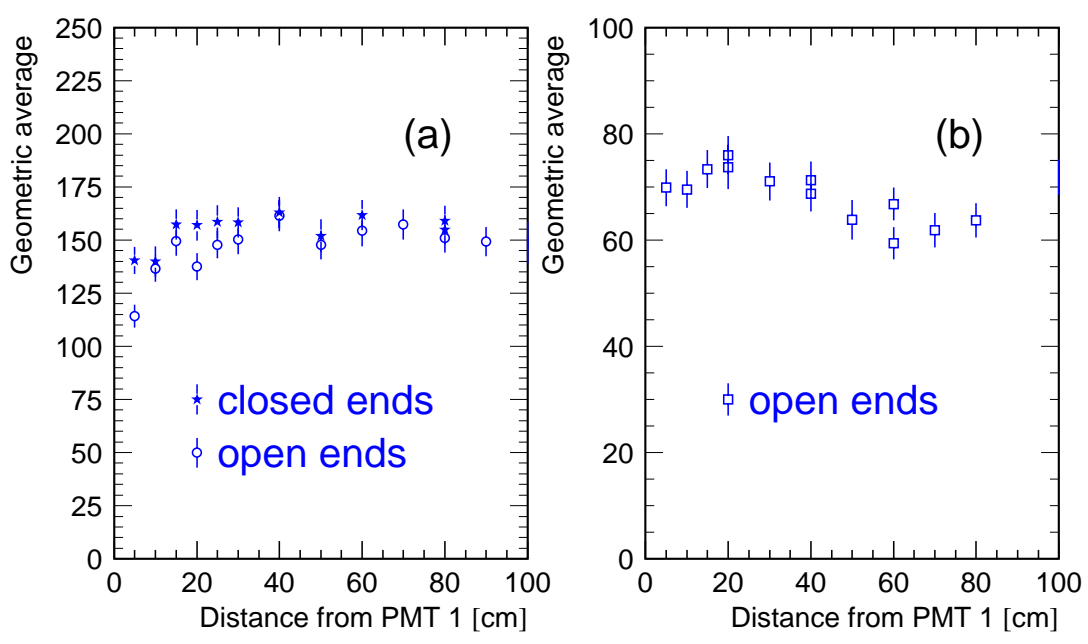

Figure 3: Comparison of the edge effect for the painted tube (a) and for the tube with the aluminium inner surface (b). For some positions, two separate measurements have been done. 

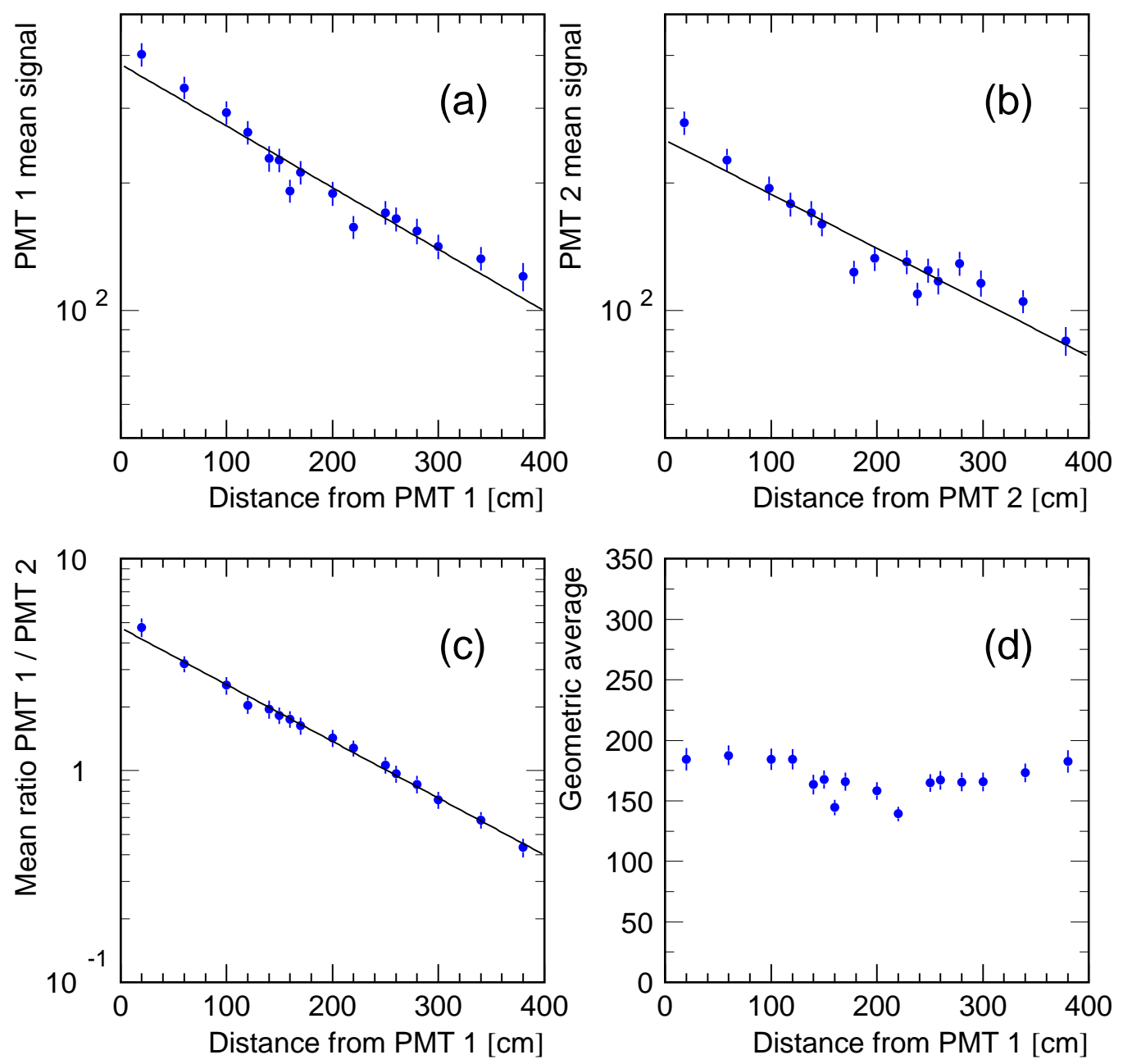

Figure 4: Measurements obtained with the $3 \times 3 \mathrm{~cm}^{2}$ aluminium tube, with the fibre directly in the liquid (Configuration 1). The average numbers of ADC counts are shown for both PMTs ( $a$ and b), as well as the average ratio (c) and the mean geometric average (d). 

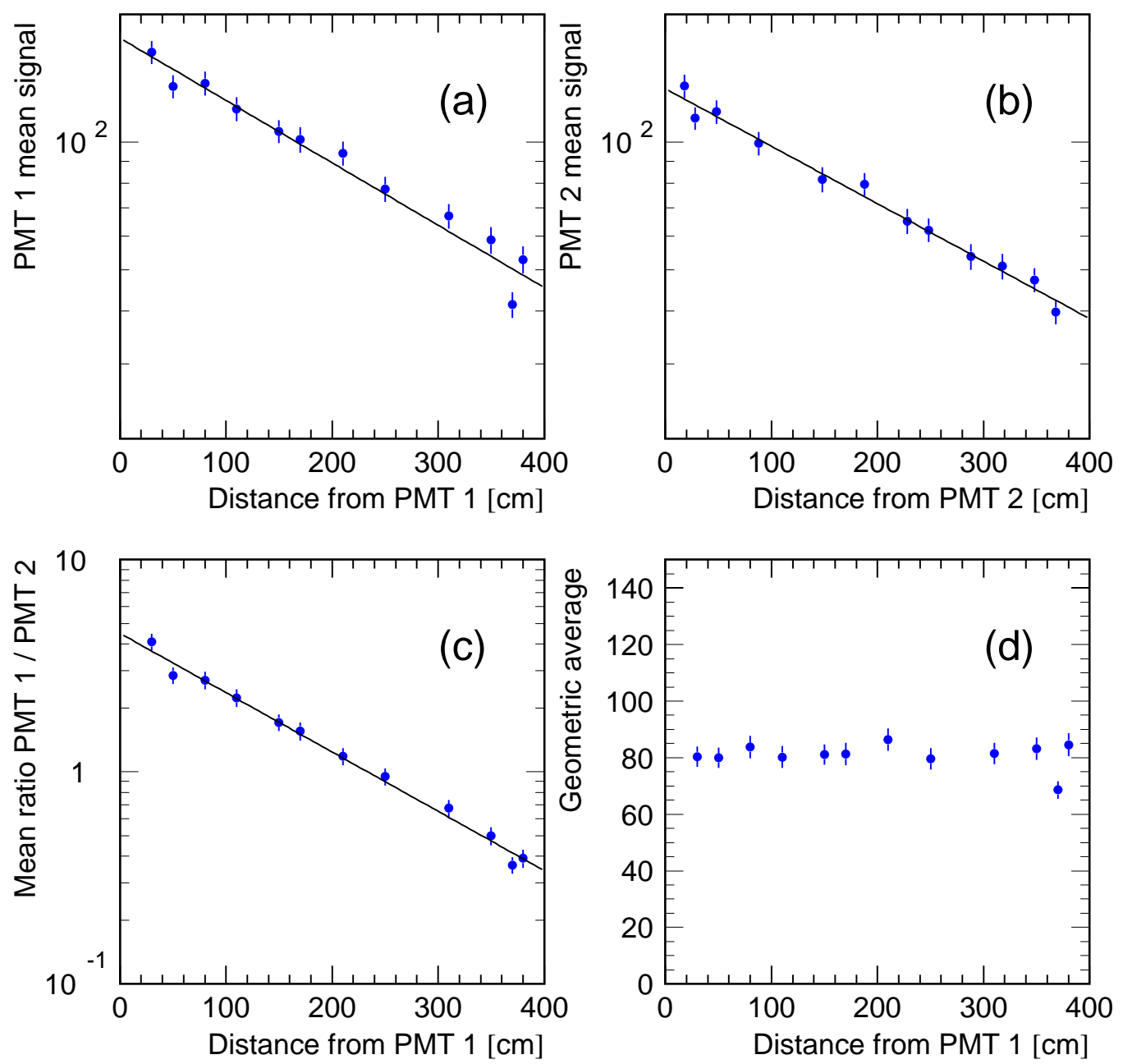

Figure 5: Measurements obtained with the $3 \times 3 \mathrm{~cm}^{2}$ aluminium tube, with the fibre in a teflon tube (Configuration 3). The average number of ADC counts are shown for both PMTs ( $a$ and b), as well as the average ratio (c) and the mean geometric average (d). 

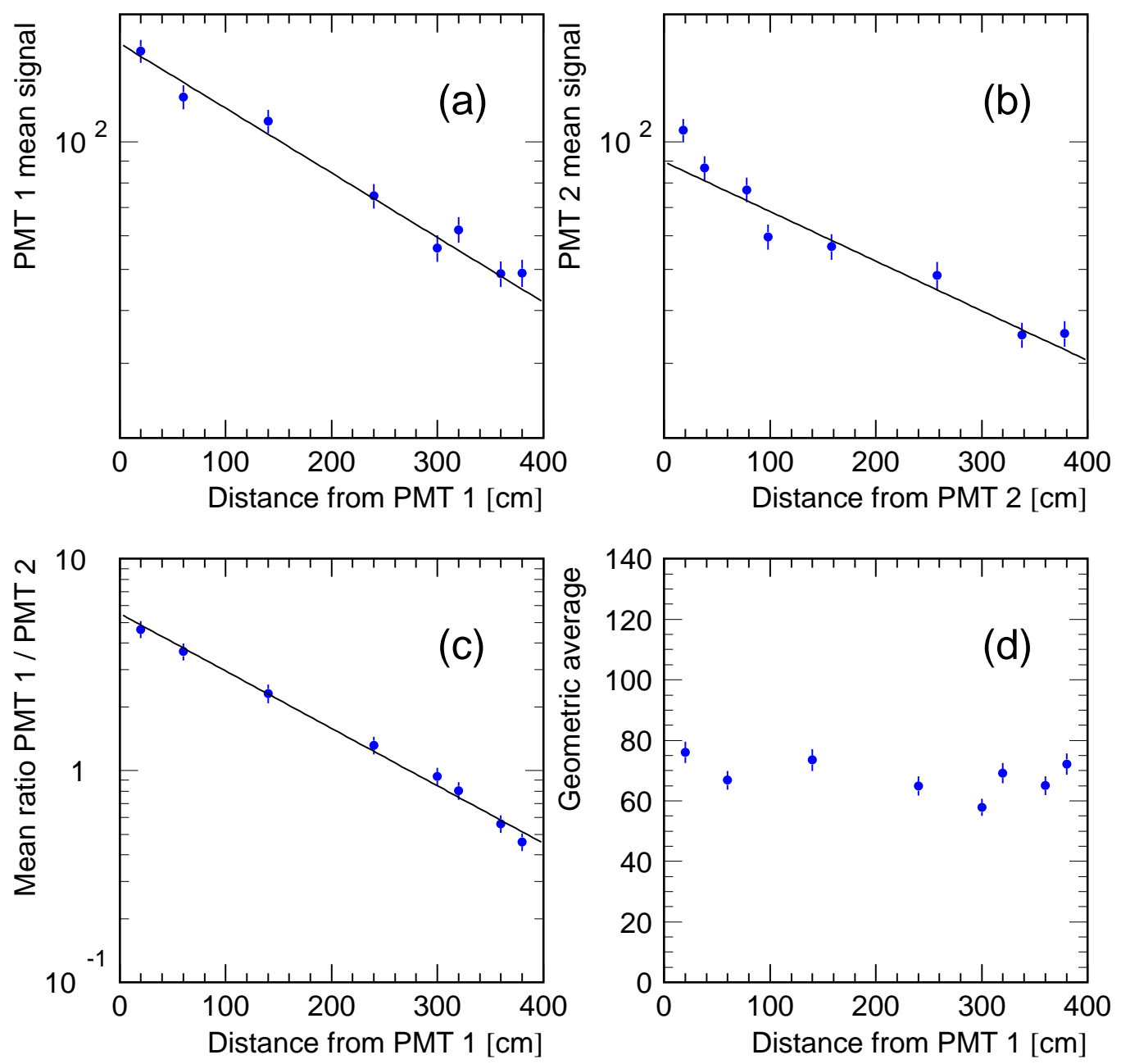

Figure 6: Measurements obtained with the $4 \times 4 \mathrm{~cm}^{2}$ aluminium tube, with the fibre directly in the liquid (Configuration 4). The average number of ADC counts are shown for both PMTs ( $a$ and b), as well as the average ratio (c) and the mean geometric average (d). 

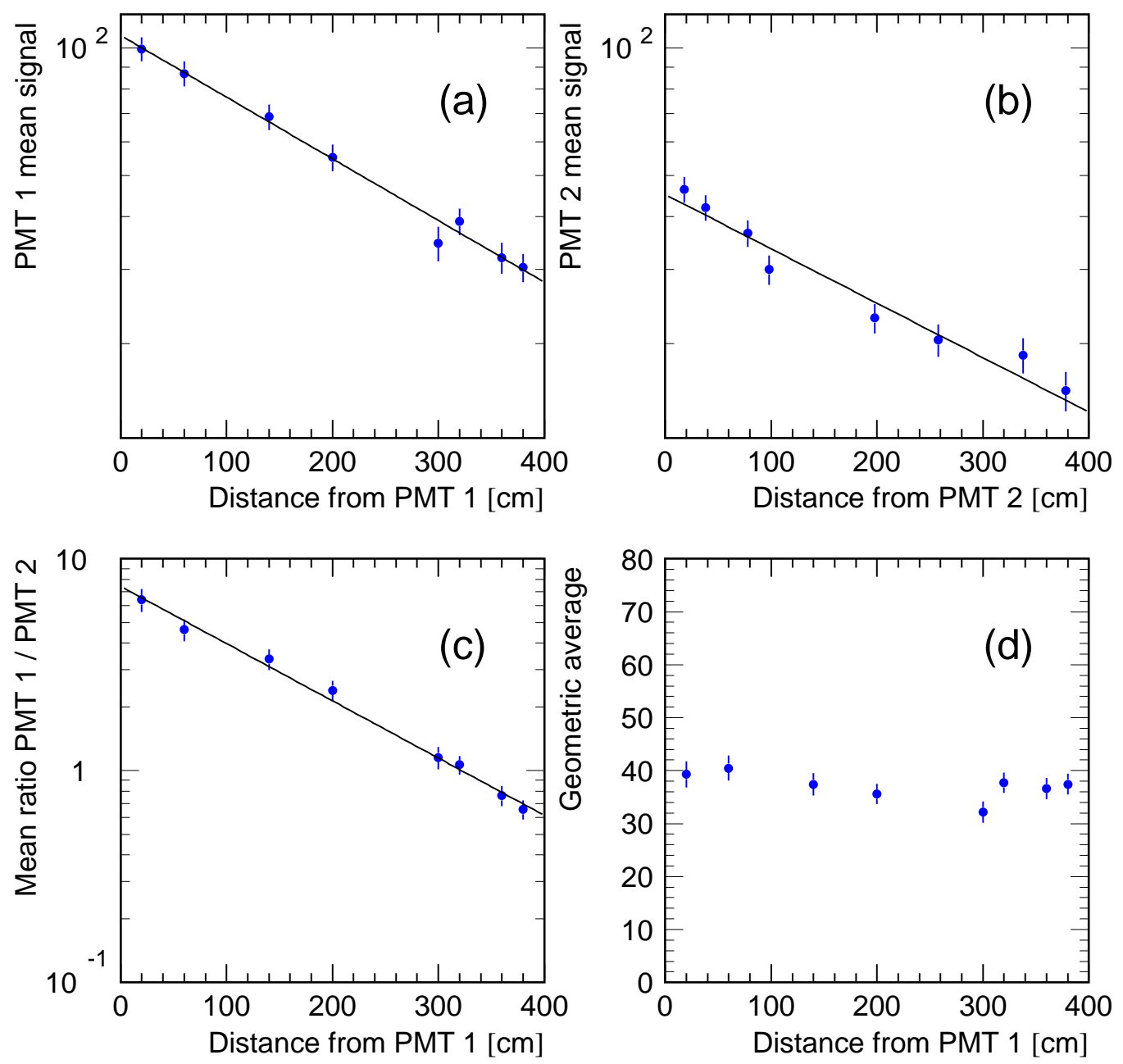

Figure 7: Measurements obtained with the $4 \times 4 \mathrm{~cm}^{2}$ aluminium tube, with the fibre in a teflon tube (Configuration 5). The average number of ADC counts are shown for both PMTs ( $a$ and b), as well as the average ratio (c) and the mean geometric average (d). 


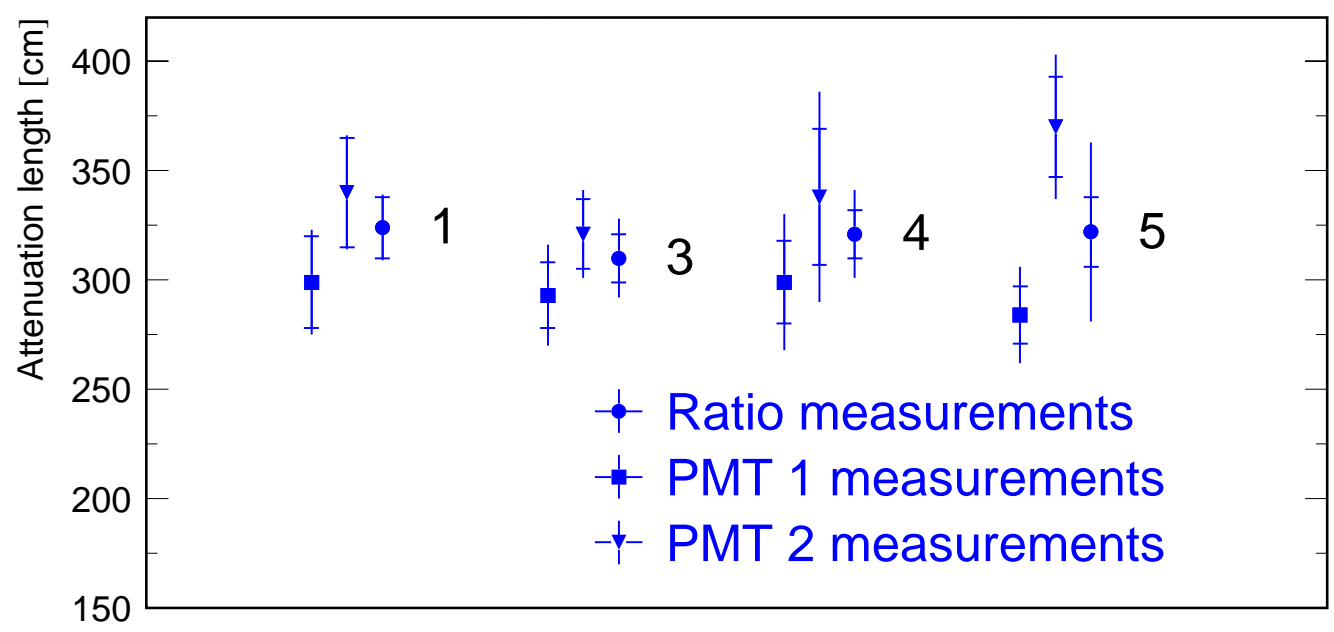

Measurements

Figure 8: Measurements of the attenuation length in different configurations. The configuration number is indicated to the right of the data points. The errors shown are both statistical (inner error bars) and systematic (outer error bars). 